\title{
Characteristics of binge eating disorder in relation to diagnostic criteria
}

\author{
This article was published in the following Dove Press journal: \\ Neuropsychiatric Disease and Treatment \\ 29 August 2016 \\ Number of times this article has been viewed
}

\author{
Denise E Wilfley' \\ Leslie Citrome ${ }^{2}$ \\ Barry K Herman ${ }^{3}$ \\ 'Department of Psychiatry, \\ Washington University School of \\ Medicine, St Louis, MO, ${ }^{2}$ Department \\ of Psychiatry \& Behavioral Sciences, \\ New York Medical College, Valhalla, \\ NY, ${ }^{3}$ Global Medical Affairs, Shire, \\ Lexington, MA, USA
}

\begin{abstract}
The objective of this review was to examine the evidentiary basis for binge eating disorder (BED) with reference to the Diagnostic and Statistical Manual of Mental Disorders - Fifth Edition (DSM-5) diagnostic criteria for BED. A PubMed search restricted to titles and abstracts of English-language reviews, meta-analyses, clinical trials, randomized controlled trials, journal articles, and letters using human participants was conducted on August 7, 2015, using keywords that included "binge eating disorder," DSM-5, DSM-IV, guilt, shame, embarrassment, quantity, psychological, behavior, and "shape and weight concerns." Of the 257 retrieved publications, 60 publications were considered relevant to discussions related to DSM-5 diagnostic criteria and were included in the current review, and 20 additional references were also included on the basis of the authors' knowledge and/or on a review of the reference lists from relevant articles obtained through the literature search. Evidence supports the duration/frequency criterion for BED and the primary importance of loss of control and marked distress in identifying individuals with BED. Although overvaluation of shape/ weight is not a diagnostic criterion, its relationship to the severity of BED psychopathology may identify a unique subset of individuals with BED. Additionally, individuals with BED often exhibit a clinical profile consisting of psychiatric (eg, mood, obsessive-compulsive, and impulsive disorders) and medical (eg, gastrointestinal symptoms, metabolic syndrome, and type 2 diabetes) comorbidities and behavioral profiles (eg, overconsumption of calories outside of a binge eating episode and emotional eating). Future revisions of the BED diagnostic criteria should consider the inclusion of BED subtypes, perhaps based on the overvaluation of shape/ weight, and an evidence-based reassessment of severity criteria.
\end{abstract}

Keywords: binge eating disorder, diagnosis, obesity, psychotherapy, pharmacotherapy, psychopathology

\section{Introduction}

As of May 2013, the Diagnostic and Statistical Manual of Mental Disorders - Fifth Edition (DSM-5) ${ }^{1}$ recognized binge eating disorder (BED) as a distinct eating disorder. To be diagnosed with BED according to DSM-5 criteria, there must be recurrent binge eating episodes (occurring on average at least once a week for $\geq 3$ months) characterized by the consumption of larger amounts of food in a discrete period than is typical for most people under similar circumstances and a sense of loss of control over eating during these episodes, and there must also be marked distress associated with the binge eating behavior. ${ }^{1}$ Unlike bulimia nervosa $(\mathrm{BN})$ and anorexia nervosa of the binge eating/purging type (AN-BE/P), there are no recurrent inappropriate compensatory behaviors, such as excessive exercise or purging, with BED. ${ }^{1}$ The DSM-5 also delineates severity criteria for BED, with the minimum level of severity based on the number of weekly binge eating episodes (mild, 1-3; moderate, 4-7; severe, 8-13;
Correspondence: Denise E Wilfley Department of Psychiatry, Washington University School of Medicine, 660 South Euclid, Campus Box 8134, St Louis, MO 63II0, USA

Tel + I 3 | 42862079

$\mathrm{Fax}+|3| 42862091$

Email wilfleyd@psychiatry.wustl.edu
Neuropsychiatric Disease and Treatment 2016:12 22/3-2223

2213

Dovepress f in 0

http://dx.doi.org/10.2147/NDTS107771 (c) (1) () ๑ 2016 Wilfey et al. This work is published and licensed by Dove Medical Press Limited. The full terms of this license are available at https://www.dovepress.com/terms.php cc. hereby accept the Terms. Non-commercial uses of the work are permitted without any further permission from Dove Medical Press Limited, provided the work is properly attributed. For permision for commercial use of this work, please see paragraphs 4.2 and 5 of our Terms (https://www.dovepress.com/terms.php). 
and extreme, $\geq 14$ ); severity level can be increased to reflect other symptoms and functional disability. ${ }^{1}$

BED was recognized as a clinical condition as early as 1959. ${ }^{2}$ However, it first appeared in the $D S M-I V^{3}$ as a provisional diagnosis that required further study. Thus, individuals meeting the criteria for BED were diagnosed with an eating disorder not otherwise specified. ${ }^{3}$ Subsequent research has established BED as a distinct eating disorder, which led to its inclusion in the DSM-5. ${ }^{1}$

Given the recognition of BED in the DSM-5 as a distinct eating disorder and the fact that the DSM-5 criteria do not differ substantially from the provisional criteria originally proposed in the $1990 \mathrm{~s}, 4,5$ it is worth reviewing the literature supporting this diagnosis to better understand the strengths and limitations of the current diagnostic criteria. This is particularly important because the estimated lifetime prevalence of BED in the US based on DSM-IV criteria (1.9\%-2.8\%) exceeds that of both BN $(\sim 1.0 \%)$ and $\mathrm{AN}$ $(\sim 0.6 \%))^{6,7}$ An improved understanding of the basis for the BED diagnostic criteria will be useful information for future $D S M$ revisions and potentially benefit treatment selection and/or predict treatment response.

This qualitative review focuses on describing the characteristics of BED with reference to DSM-5 diagnostic criteria and highlights the aspects of the DSM-5 diagnostic criteria requiring further consideration, including the definition of what constitutes a "large amount of food" with reference to binge eating, and the lack of inclusion of overvaluation of shape and weight as a diagnostic criterion. Thus, this review summarizes the evidentiary basis for the DSM-5 BED diagnostic criteria; the factors that differentiate BED from other eating disorders and obesity; and the comorbidities, psychopathologies, functional disabilities, and impaired quality of life (QoL) associated with BED. Based on this evidence, a discussion on the limitations of the current diagnostic criteria and recommendations for future research requirements are provided.

\section{Methods}

\section{Literature review}

A PubMed search restricted to titles and abstracts of Englishlanguage systematic reviews, meta-analyses, clinical trials, randomized controlled trials, journal articles, and letters that used human participants was conducted on August 7, 2015. Search terms used were ("binge eating disorder" OR "binge eating") AND (DSM-5 OR DSM-IV) AND ("loss of control" OR guilt OR shame OR embarrassment OR quantity OR treat OR treatment OR psychological OR behavior OR "shape and weight concerns" OR "shape/weight concerns" OR "body image" OR "shape and weight overvaluation" OR "shape/weight overvaluation" OR severity OR specifier OR subtyping OR “binge eating frequency” OR comorbid OR comorbidities). Although BED can be identified in children and adolescents, ${ }^{8,9}$ only a few meet all symptom criteria for BED. Therefore, the current review focuses only on the adult literature. It should also be noted that shape and weight concerns are not currently included in the diagnostic criteria for BED. However, it has been suggested that they should be included as a diagnostic specifier or can be used for dimensional rating of severity in BED. ${ }^{10}$ As such, this term was included in the literature search so that the evidence base for this topic could be reviewed. Additional references were included based on the authors' knowledge of relevant articles and on a review of the reference lists from relevant articles obtained through the literature search.

\section{Results}

\section{Summary of search results}

A total of 257 publications were retrieved through the PubMed search. Of these, 197 were eliminated as being irrelevant to the objectives of this study based on a review of the published abstracts, and the remaining 60 publications were considered relevant to discussions related to DSM-5 diagnostic criteria and were included in the current review. An additional 20 references were also included based on the authors' knowledge of relevant publications and/or on a review of the reference lists from relevant articles obtained through the literature search.

\section{Evidence base for DSM-5 BED diagnostic criteria}

There is variability in the amount of evidence available for the individual diagnostic criteria for BED. However, in a study that examined the heritability of BED, factor loadings for each of the diagnostic criteria for BED were found to be highly associated with the propensity toward BED. This finding indicates that each of the BED diagnostic criterion is related to a unidimensional construct (ie, a single psychiatric diagnosis). ${ }^{11}$ The following sections describe the supportive evidence for specific BED diagnostic criteria, highlight the limitations of some of these criteria, and discuss the clinical phenotypes that are not included in the DSM-5 BED criteria.

\section{Amount of food consumed}

Although the BED diagnostic criteria refer to objective binge eating episodes (eg, eating a larger amount of food in 
a discrete period than what a typical person would consume under similar circumstances and eating a large amount of food when not physically hungry), ${ }^{1}$ the role of subjective binge eating episodes (eg, eating what is "perceived" by that person to be a large amount of food even if it is not an amount that is typically considered large $)^{12-14}$ is not addressed in the DSM-5 BED diagnostic criteria. Individuals who report only having subjective binge eating episodes do not meet $D S M-5$ diagnostic criteria for BED. Some justification for excluding subjective binge eating episodes from the BED diagnostic criteria can be derived from the fact that no published comparative data on subjective binge eating episodes in individuals with versus without BED are available. In contrast, the frequency of objective binge eating episodes has been shown to be higher in obese individuals with BED than in obese individuals without BED. ${ }^{15,16}$ Furthermore, the limited available data on subjective binge eating episodes in individuals with BED or in those meeting partial criteria for BED indicate that there are few meaningful differences in binge eating severity or psychopathology when individuals exhibiting subjective versus objective binge eating episodes are compared. ${ }^{12,14}$ To more clearly delineate the relative importance of subjective versus objective binge eating episodes in BED, systematic assessment of the proportion of individuals with DSM-5-defined BED who report subjective binge eating episodes and the relative proportions of subjective versus objective binge eating episodes reported by these individuals needs to be carried out.

In further regard to the amount of food consumed in $\mathrm{BED}$, it is also difficult to definitively state what constitutes "an amount of food that is definitely larger than what most people would eat during a similar time period under similar circumstances" because this definition is based on clinical judgment, which varies across health care professionals. The fact that obese individuals with BED often overconsume food relative to obese individuals without BED during the periods of normal consumption also complicates this issue. ${ }^{17-19}$ In conclusion, there is a degree of uncertainty about what constitutes a "large amount of food" with reference to binge eating and, perhaps due to the limited available data on individuals with BED, whether subjective binge eating should be considered in the BED diagnostic criteria.

\section{Loss of control}

Among the diagnostic criteria for BED, a sense of loss of control over eating has been shown to be foundational to the BED diagnosis. ${ }^{20-26}$ In a study on obese females being assessed for bariatric surgery, the frequency of reporting loss of control over eating was higher in participants who selfreported binge eating (75\%) than in those who self-reported only overeating $(22 \%){ }^{26}$ In another study that examined BED diagnostic criteria using the Questionnaire of Eating and Weight Patterns, loss of control over eating was reported in $84 \%$ of the self-referred individuals who reported binge eating or compulsive overeating to be problematic compared with $35 \%$ of controls who did not. ${ }^{25}$ The odds of experiencing loss of control over eating were 3.6 times greater in individuals with BED than in individuals without BED after controlling for affective state and caloric intake, which suggests that loss of control is an inherent component of BED. ${ }^{20}$ In support of this conclusion, when females with BED were asked to define binge eating, $82 \%$ included loss of control. ${ }^{21}$ In this same study, responses of the type "feeling totally out of control" and "a binge is not being able to stop eating or stop before it's all gone" were typical across participants. ${ }^{21}$ However, at least one study that compared treatment-seeking obese females with BED to obese females without BED (but did not report a loss of control regarding their eating) did not report consistent differences in eating symptoms or general psychopathology between these groups. ${ }^{27}$ The authors suggested that the lack of differentiations in this study could partly be due to the fact that all participants in the study were treatment-seeking and may have been exhibiting higher-level eating disorders and psychopathology than non-treatmentseeking individuals who are obese.

\section{Binge eating episode characteristics}

There is limited evidence in the published literature to support the individual characteristics of a binge eating episode described in the DSM-5. Of the five indicators of impaired control listed in the DSM-5 (ie, determinants of loss of control during a binge eating episode), eating large amounts of food when not hungry and eating alone because of embarrassment were found to be the best predictors of a BED diagnosis. ${ }^{22}$

\section{Marked distress}

The published literature indicates that BED is associated with general psychiatric distress ${ }^{15,28-30}$ and, more specifically, with marked distress in regard to binge eating. . $^{211,23,29,31-34}$ As early as 1959, case reports of Stunkard indicated that marked distress was reported in people who binge eat. ${ }^{2}$ High levels of distress related to binge eating are reported in individuals with BED who are normal weight and in individuals with BED who are obese, suggesting that the distress is not simply a consequence of comorbid obesity. ${ }^{33}$ The integral nature of binge eating-related distress in individuals with BED is 
supported by genetic studies, which indicate that marked distress is a heritable component of BED. ${ }^{11}$

Individuals with BED demonstrating marked distress related to binge eating exhibit increased levels of eating disorder pathology and depressive symptoms compared with individuals meeting all BED criteria except marked distress and with individuals who are obese but do not exhibit binge eating or purging behavior. ${ }^{31}$ Furthermore, levels of distress associated with binge eating in individuals with BED have been reported to differ as a function of the presence of overvaluation of shape and weight, which is associated with increased distress, ${ }^{32}$ and race/ethnicity. Although distress is reported across all examined races/ethnicities, higher levels of distress associated with binge eating are more likely to be reported in individuals who are Latino or Caucasian than in individuals who are Asian or African-American. ${ }^{34}$ Therefore, although the level of distress may vary across subpopulation, the overall literature provides support for the legitimacy of the DSM-5 marked distress criterion for BED. ${ }^{2,11,23,29,31-34}$

\section{Binge eating frequency and duration}

Although there was controversy regarding the binge eating frequency criterion in the DSM-IV - text revision (DSM-IV-TR; ie, at least twice a week for 6 months $\left.{ }^{35}\right)$, which was chosen to set a high threshold for diagnosing BED in the absence of empirical support for a less stringent criterion, ${ }^{36}$ the currently available data support the less stringent criterion (ie, at least once a week for 3 months $^{1}$ ) of the DSM-5. ${ }^{29,37-40}$ For instance, females with subthreshold BED (defined by a binge eating frequency of at least once a month for 6 months) exhibited eating disorder patterns and psychiatric symptoms, with the exception of shape concern, similar to those of females with full-syndrome BED (as defined by DSM-IV-TR criteria). ${ }^{29}$ Furthermore, individuals with subthreshold BED (binge eating episodes less than twice a week but at least once per 28 days or eating $<800 \mathrm{kcal}$ per binge at least twice per week) did not differ in eating psychopathology, as measured by the dietary restraint, disinhibition, and hunger dimensions of the Three-Factor Eating Questionnaire, from those meeting full DSM-IV-TR BED criteria. ${ }^{38}$

It has been suggested that the revised frequency/ duration criterion for BED in the DSM-5 will increase the ability to detect binge eating pathology without markedly changing lifetime prevalence, ${ }^{40}$ which would be indicative of increased diagnostic sensitivity (ie, the percentage of individuals with BED correctly identified as having this condition would increase). A study estimated that the
$D S M$-5 BED criteria would produce a relative increase in the lifetime prevalence of $\sim 3 \%$, with the resulting total lifetime prevalence of BED in the United States increasing to $3.6 \%$ in females and $2.1 \%$ in males. ${ }^{39}$ Another study estimated that lifetime prevalence would increase by $0.18 \%$ when the frequency/duration criterion shifted from $\geq 8$ times per month for 6 months (the DSM-IV-TR criterion) to $\geq 4$ times per month for 3 months (as this study was conducted before the release of the DSM-5 BED criteria, it utilized the approximate $D S M-5$ frequency criterion that later became the actual criteria). ${ }^{40}$ Based on the available evidence, the less stringent binge eating frequency/duration criterion for BED used in the DSM-5 is likely to increase the sensitivity of BED diagnosis (ie, ensure that individuals with BED are properly diagnosed) without markedly changing its specificity (ie, ensure that individuals without BED are not diagnosed) relative to the $D S M-I V-T R$ provisional criteria. Overall, the available evidence supports the less stringent frequency/duration criteria of the DSM-5 compared with the provisional criteria of the $D S M-I V-T R,{ }^{29,37-40}$ even though the exact criteria of the DSM-5 have not yet been specifically validated.

\section{Differentiating BED from BN or AN}

The DSM-5 diagnostic criteria for BED are intended to identify individuals who have significant eating pathologies that are distinct from those observed in other eating disorders. ${ }^{24}$ Diagnostically, BED is distinguished from $\mathrm{BN}$ and from $\mathrm{AN}-\mathrm{BE} / \mathrm{P}$ by the lack of recurrent compensatory behaviors in $\mathrm{BED}$, which are necessary for the diagnosis of $\mathrm{BN}$ and may be present in AN-BE/P but are not required. ${ }^{1}$ In addition, DSM-5 diagnostic criteria for BED do not include shape and weight concerns, which are included in the criteria for both $\mathrm{AN}$ and BN. ${ }^{1}$ However, it should be reiterated that a substantial proportion of individuals with BED report shape and weight concerns. ${ }^{10,30,32,41}$ Perhaps because of these diagnostic distinctions, lifetime diagnoses of $\mathrm{AN}$ or $\mathrm{BN}$ in individuals who also meet BED criteria have generally been reported to be low (1.5\% and 5.9\%, respectively) in adults. ${ }^{42}$ However, it should also be noted that diagnostic crossover was reported to be higher (BN to BED [23\%] and BED to BN [20\%]) in a community sample of adolescent females, ${ }^{43}$ suggesting that transitions between $\mathrm{BN}$ and BED may be more common prior to adulthood.

$\mathrm{BED}$ differs from $\mathrm{AN}$ and $\mathrm{BN}$ in terms of prevalence, age of onset, and eating behaviors and pathologies. . $7,10,16,31,44-46^{-1}$ The relative lifetime prevalence of BED in both males and females $(2.0 \%$ in males and $3.5 \%$ in females $)$ is higher than 
that observed in $\mathrm{BN}(0.5 \%$ vs $1.5 \%)$ or $\mathrm{AN}(0.3 \%$ vs $0.9 \%){ }^{6}$ $\mathrm{BED}$ also differs from $\mathrm{BN}$ in that it has a later age of onset (mean age, 23.3 vs 20.6 years; interquartile range for age of onset, 15.5-27.2 years for BED and 14.5-22.9 years for $\mathrm{BN}$ ) and may have a lower persistence (median years in episode, 4.3 vs 6.5$){ }^{7}$ although others propose that the course is comparable. ${ }^{1}$ In individuals with BED, scores on measures of dietary and cognitive restraint are lower than that in individuals with $\mathrm{BN}$. $^{10,16,31,44}$ Individuals with BED also exhibit a significantly lower frequency of binge eating episodes than do individuals with BN. ${ }^{10,16}$ However, unlike individuals with $\mathrm{BN}$, individuals with $\mathrm{BED}$ overconsume food during both binge eating episodes and non-binge eating episodes. ${ }^{45,46}$ Overall, $, 6,10,16,31,44-46$ there is clear evidence that $\mathrm{BED}$ is distinct from $\mathrm{AN}$ and $\mathrm{BN}$.

\section{Binge eating disorder severity criteria}

The DSM-5 provides severity criteria for BED, with the minimum level of severity based on the number of weekly binge eating episodes (mild, 1-3; moderate, 4-7; severe, 8-13; and extreme, $\geq 14$ ), and the severity level can be increased to reflect other symptoms and functional disability. ${ }^{1}$ As of the release of the DSM-5 in 2013, there was no empirical evidence to support the recommended BED severity criteria of the DSM-5. In two studies published in 2015, the BED severity categories of the DSM-5 were evaluated in clinical and community populations of adults diagnosed with BED. ${ }^{47,48}$ The percentages of participants in these studies (clinical population vs community population) categorized as having BED of mild, moderate, severe, and extreme severity based on DSM-5 criteria were $39.7 \%$ versus $78.1 \%$, $47.4 \%$ versus $19.8 \%, 10.0 \%$ versus $1.8 \%$, and $3 \%$ versus $0.3 \%$, respectively, ${ }^{47,48}$ indicating that the clinical population exhibited more severe BED symptoms as would be expected. When the BED severity groups in the clinical population were compared across clinical characteristics (Eating Disorder Examination [EDE] Questionnaire global and subscale scores and Beck Depression Inventory scores), eating pathology significantly increased as a function of BED severity on three EDE subscales (ie, eating concern, shape concern, and weight concern) and on Beck Depression Inventory score. ${ }^{48}$ In the community population, increased BED severity was associated with increased EDE global and subscale scores. ${ }^{47}$ Based on these data, the categorization of BED severity as a function of weekly binge eating episodes appears valid. However, data providing support for the modification of BED severity based on other symptoms or functional disability are not available.

\section{Evidence base for factors not included as diagnostic criteria Obesity}

Although obesity is associated with BED, it is not included as a diagnostic criterion for BED and the DSM-5 indicates that BED is distinct from obesity. ${ }^{1}$ BED is reported across body mass index (BMI) categories but is most common in obese individuals $(36.2 \%-42.4 \%) .{ }^{6,7}$ A substantial minority of individuals seeking weight loss treatment also have a BED diagnosis (13\%-27\%). ${ }^{28,49-51}$ Among obese individuals, those meeting the DSM-5 BED diagnostic criteria are a meaningful and distinct subgroup. Obese individuals with BED have poorer psychological functioning (eg, higher depression, lower self-esteem, and more emotional eating and shape and weight concerns) than obese individuals without BED. ${ }^{15,16,50,52}$ In addition, compared with obese individuals without BED, obese individuals with BED exhibit distinct eating behaviors beyond binge eating. For instance, obese individuals with BED consume more calories than obese individuals without BED when asked to binge eat in a laboratory setting ${ }^{17-19,53}$ and when asked to eat a normal (ie, nonbinge) meal in a laboratory setting. ${ }^{18,19}$ These findings raise the unanswered question of why obese individuals with BED do not have substantially higher BMI than obese individuals without BED because individuals with BED consume more calories overall and have similarly low levels of physical activity. ${ }^{54}$

The concept that BED is a phenotype that is distinct from obesity is further validated by genetic studies. ${ }^{11,55,56}$ These studies indicate that BED is heritable, ${ }^{11,55,56}$ that BED clusters in families independent of the presence of obesity, and that individuals with a family history of BED are at greater risk of obesity in adulthood than those with a family history of obesity but not with BED. ${ }^{56}$ Overall, the literature emphasizes that there are distinct differences between obese individuals with and without BED. ${ }^{15-19,50,52,53}$

\section{Overvaluation of shape and weight}

There has been controversy regarding the role of overvaluation of shape/weight in BED and its lack of inclusion as a diagnostic criterion for $\mathrm{BED},{ }^{24,57}$ in part due to discrepancies in the published literature. Overvaluation of shape/ weight was clinically recognized in individuals with BED in the 1990s. ${ }^{58}$ The literature suggests that overvaluation does not simply reflect obesity-related concerns or distress. Rather, independent of BMI, it is reliably associated with the severity of eating pathology, psychological distress, and negative prognostic significance $\mathrm{e}^{10,15,30,32,41,59}$ and with worse treatment outcomes ${ }^{60-62}$ in individuals with BED. However, 
a substantial proportion ( $40 \%)$ of individuals with BED do not exhibit overvaluation of shape and weight, ${ }^{10,32,41}$ in marked contrast to BN where most individuals (95\%) exhibit overvaluation. ${ }^{10}$

Despite this discrepancy, individuals with BED who exhibit clinically relevant overvaluation of shape/weight (defined as scores of $\geq 4$ on either overvaluation item of the EDE) tend to have more severe eating disorder pathology, as evidenced by higher scores on the eating concerns and dietary restraint subscales of the EDE than those who exhibit subclinical overvaluation of shape/weight (defined as scores of $<4$ on both overvaluation items of the EDE). ${ }^{10}$ Consistent with a preoccupation with shape/weight, body checking behavior (eg, frequent weighing or measuring of fat deposits) in obese females with BED is positively correlated with overvaluation of weight and depressive symptoms and is negatively correlated with self-esteem (although similar correlations were not found in males with BED). ${ }^{63}$ Overvaluation of shape/weight has also been found to be associated with psychiatric and social function impairments (eg, depression, anxiety, decreased self-esteem, and functional impairments) in individuals with BED. ${ }^{15,30,32,59}$ Individuals with BED who do not demonstrate shape/weight overvaluation have been reported not to differ substantively from obese individuals without BED in terms of eating disorder pathology and functional impairment. ${ }^{59}$

In sum, there is evidence demonstrating that overvaluation of shape/weight is strongly related to eating pathology severity in individuals with BED and that it has negative prognostic significance in individuals with BED. ${ }^{10,15,30,32,41,57,59}$ This suggests that overvaluation of shape/weight should be considered as a diagnostic specifier, which designates a subtype of BED, or a modifier that could increase the index of BED severity. If it were a required diagnostic criterion, it would result in the exclusion of a substantial number of individuals who otherwise do exhibit significant pathology and distress ${ }^{10}$ because a substantial proportion of individuals with BED do not exhibit overvaluation of shape/weight. ${ }^{10,32,41}$

\section{Comorbidities, psychopathologies, functional disabilities, and impaired QoL}

As described earlier in this review, the DSM-5 diagnostic criteria for severity based on binge eating frequency can be adjusted on the basis of the presence of other symptoms and by the degree of functional disability. ${ }^{1}$ Although there is no empirical basis for adjusting the severity based on functional disability, a substantial body of evidence (see Davis ${ }^{64}$ and Sheehan and Herman ${ }^{65}$ for recent reviews) suggests that BED is associated with psychiatric and medical comorbidities, functional disability, and impaired QoL. ${ }^{67,72,50,62,66-76}$

\section{Psychiatric comorbidities and psychopathologies}

Individuals with BED report higher rates of major depressive disorder, posttraumatic stress disorder, generalized anxiety disorder, obsessive-compulsive disorder, panic attacks, impulse control disorders, and substance abuse than individuals without BED. ${ }^{6,7,42,50,62,66-68}$ For example, the lifetime prevalence for any mood disorder and anxiety disorder among individuals with BED has been estimated to range from $46 \%$ to $54 \%$ and from $37 \%$ to $65 \%$, respectively. ${ }^{6,742}$

The relationship between BED and psychiatric comorbidities may be complex and influenced by factors that include comorbid disease severity and race. ${ }^{44,77-81}$ Among individuals with BED, the severity of depression or anxiety symptoms can influence the likelihood of binge eating. ${ }^{77}$ Similarly, emotional eating (ie, eating in response to negative emotions and anxiety) correlates with binge eating and most eating psychopathology measures in obese individuals with BED. ${ }^{78,79}$ Regarding race and ethnicity, individuals with BED who are Black or Hispanic have been found to be more than twice as likely as individuals with BED who are White to have a comorbid mood and anxiety disorder. ${ }^{80}$ Elevated psychiatric comorbidities in BED can occur independently of obesity and weight status, with studies reporting that individuals with BED who are obese and individuals with BED who are not obese have similar levels of depressive symptoms. ${ }^{44,81}$

In addition to psychiatric comorbidities, certain personality profiles and psychopathologies in individuals with BED are associated with binge eating behaviors. ${ }^{50,82}$ In a study of obese females with BED, binge eating severity was correlated with paranoid ideas, psychoticism, and interpersonal sensitivity. ${ }^{50}$ Furthermore, individuals with BED exhibit interpersonal problems, which are negatively correlated with age at first binge, and less flexible interpersonal styles, which increase the likelihood of meeting BED diagnostic criteria at a young age. ${ }^{82}$

Taken together, this is a substantial evidence base indicating that BED is associated with increased psychiatric comorbidity and psychopathology and distinctive personality profiles. ${ }^{44,50,77-82}$ It is important for clinicians to be aware of these comorbid conditions, because they may be the manifest reasons that individuals with BED seek treatment, particularly if they are not aware that BED is an actual disorder. Furthermore, the personality profiles, presence of specific comorbidities, and their severity may influence treatment strategies. 
Nonpsychiatric comorbidities and medical profiles

BED is associated with nonpsychiatric medical comorbidities that include sleep disturbances, type 2 diabetes, metabolic syndrome, and gastrointestinal distress. ${ }^{69-73} \mathrm{~A}$ large percentage of individuals with BED $(>40 \%)$ meet the criteria for metabolic syndrome. ${ }^{69,70}$ Consistent with this finding, in a 5-year longitudinal study, individuals with BED who were obese were found to be at increased risk for developing components of metabolic syndrome compared with individuals without BED who were BMI-matched to those with BED. ${ }^{74}$ In a study of weight loss surgery candidates, $\sim 8 \%$ of individuals met diagnostic criteria for both type 2 diabetes and BED, with black race and male sex being strong indicators of the co-occurrence of these disorders. ${ }^{71}$

\section{Functional disability and QoL}

Data on QoL and functional disability in individuals with BED are relatively limited, but publications to date do support the concept that BED is associated with impaired QoL and with increased functional disability. ${ }^{7,73,75,76,83}$ Based on the Medical Outcomes 36-item Study Short-Form Health Survey, all domains of health-related QoL are impaired in individuals with BED relative to the normative US population and on the emotional role limitation, mental health, and social functioning domains relative to obese individuals without BED. ${ }^{76}$ Furthermore, in a study of females conducted at primary care and gynecologic clinics throughout the US, BED was associated with worse health-related QoL on all domains of the Medical Outcomes 20-item Study Short-Form Health Survey compared with females without psychiatric disorders. ${ }^{73}$ In regard to functional disability, BED is associated with impaired role attainment relative to individuals without BED as measured by females with BED (but not males) being less likely to marry and males with BED (but not females) being less likely to be employed. ${ }^{75}$ On the Sheehan Disability Scale, $\sim 50 \%$ of individuals with BED reported some level of role impairment. ${ }^{7}$ In a randomized, placebo-controlled study of topiramate, ${ }^{83}$ mean Sheehan Disability Scale total scores at baseline ( $\sim 12$ points) were indicative of mild-to-moderate functional impairment. ${ }^{84}$

\section{Limitations of the DSM-5 diagnostic criteria for BED}

The DSM-5 diagnostic criteria for BED are intended to identify a population of individuals whose psychopathologic and behavioral profiles are distinct from other eating disorders and obesity. However, there are several limitations of the current criteria and outstanding issues that remain to be addressed. The strongest evidence supporting the DSM-5 diagnostic criteria is available for the loss of control, ${ }^{20-24}$ marked distress, ${ }^{2,11,23,29,31-34}$ and the frequency/duration criteria. $^{29,37-40}$ Although the presence of loss of control over eating in BED is empirically supported in the published literature, ${ }^{20-24}$ the role of subjective binge eating episodes in BED has not been extensively studied, and what constitutes consuming an amount of food that is "definitely larger than what most people would eat under similar circumstances..." during an objective binge eating episode is not well defined and is based on clinical judgment.

An index of BED severity is included in the $D S M-5$, which establishes a minimum level of severity based on the number of binge eating episodes per week (mild, 1-3; moderate, 4-7; severe, 8-13; and extreme, $\geq 14$ ) and allows for increases in severity based on the presence of comorbidities and functional disabilities. ${ }^{1}$ This represents an expansion of the criteria compared with the DSM-IV-TR, in which severity criteria were not included. ${ }^{35}$ Although there is some evidence supporting the new severity criterion based on the number of weekly binge eating episodes, ${ }^{47,48}$ the degree to which comorbidities, other symptoms, or functional disability influence severity is not clear. Furthermore, the impact of the method that is used to assess symptom severity needs to be clarified because symptom level could vary between patient self-report versus physician-administered clinical interview. In addition, a systematic review reported that some self-reported binge eating measures exhibit better psychometric properties than others but that further study is required in terms of the validity and reliability of most binge eating measures. ${ }^{85}$

Overvaluation of shape/weight is not addressed in the $D S M-5$, despite the fact that it has been recognized by researchers as an important feature of BED. ${ }^{24,57}$ Overvaluation of shape/weight is known to have prognostic value for $\mathrm{BED}^{32,57}$ and is included as part of the diagnostic criteria for $\mathrm{BN}$ and AN. ${ }^{1}$ Although there is empirical evidence for not including overvaluation of shape/weight as a required criterion for $\mathrm{BED},{ }^{10}$ its total exclusion from the DSM-5 criteria is not warranted based on its prognostic value and association with the severity of eating disorder pathologies in individuals with BED. ${ }^{10,15,30,32,41,57,59}$ Using the overvaluation of shape and weight to designate a distinct subtype of BED or as a modifier of BED severity should be taken into consideration.

Although individuals meeting $D S M-5$ criteria for BED share commonalities (eg, more likely to be overweight, to be female, and to have elevated anxiety and/or depression), there are important individual differences among individuals with BED. Future research should examine the utility 
of including distinct subtypes of BED in the diagnostic criteria. In support of this recommendation, a factor analysis from one study identified two BED subtypes (a pure dietary subtype and a negative affect BED subtype), with the latter exhibiting a higher frequency of binge eating episodes and sensitivity to punishment, as measured by the Sensitivity to Punishment and Sensitivity to Reward Questionnaire. ${ }^{86}$ Another analysis identified four distinct BED classes (Class 1: increased physical activity levels and lower BMI; Class 2: high levels of binge eating, shape/weight concerns, compensatory behaviors, and negative affect; Class 3: high levels of binge eating with low levels of exercise or compensation; and Class 4: high BMI, frequent overeating with fewer binge episodes, and no compensatory behavior). ${ }^{87}$ Of note, the potential existence of a BED subtype characterized by compensatory behavior complicates the current diagnostic guidelines and renders indistinct the differences between BED and other eating disorders. Lastly, although individuals with BED who are not obese have been reported to be similar to individuals with BED who are obese in many ways, ${ }^{44,81}$ it has been reported that individuals with BED who are not obese may display more dietary and cognitive restraint than individuals with BED who are obese. ${ }^{81}$ Although not all studies have replicated this finding, ${ }^{44}$ the potentially increased restraint in individuals with BED who are not obese may blur the distinction between BED and nonpurging BN (ie, individuals with $\mathrm{BN}$ who compensate through dietary fasting or excessive exercising rather than purging).

\section{Conclusion}

The diagnostic entity of BED, which has been recognized in clinical settings and the research literature for $>50$ years, ${ }^{2}$ is a heterogeneous and complex disorder. Individuals diagnosed with BED often exhibit a unique clinical profile consisting of psychiatric ${ }^{6,7,42,50,62,66-68}$ (eg, mood, obsessive-compulsive, and impulsive disorders) and nonpsychiatric medical ${ }^{69-73}$ (eg, sleep disturbance, gastrointestinal symptoms, metabolic syndrome, and type 2 diabetes) comorbidities, psychopathologies ${ }^{32,57,63}$ (eg, overvaluation of shape and weight), and personality/ behavioral traits ${ }^{17-19,50,78,79,82,88}$ (eg, overconsumption of calories, emotional eating, and interpersonal problems). Because comorbid conditions may be one of the reasons individuals with BED seek treatment, physicians need to be aware of their presence. Although evidence indicates that BED is more prevalent than both $\mathrm{AN}$ and $\mathrm{BN}^{6,7}$ and is associated with worse QoL $^{73,76}$ and increased functional disability, ${ }^{7,75,83}$ BED continues to be underrecognized, underdiagnosed, and undertreated in clinical settings. ${ }^{7}$
When considering patients with putative BED, health care professionals should focus on the presence of clinical indicators and risk factors to aid in diagnosis. ${ }^{89}$ These indicators can include obesity and clinically significant weight gain $(\geq 5 \%$ of body weight) in the past year, psychiatric and medical comorbidities, and a family history of BED. There are also assessment tools that can be used by health care professionals when assessing the patients for potential BED. These include the validated seven-item binge eating disorder screener (BEDS-7), ${ }^{90}$ the validated Eating Disorder Assessment for the DSM-5, ${ }^{91}$ and the Eating disorder Screen for Primary Care (ESP). ${ }^{92}$ The ESP and Eating Disorder Assessment for the DSM-5 are limited in that they do not focus specifically on BED, as does the BEDS-7. Furthermore, the BEDS-7 and ESP are screeners that are not intended to be used as diagnostic tools; individuals who screen positive on the BEDS-7 or ESP should be referred to a specialist for a formal diagnosis.

With the release of the DSM-5 diagnostic criteria for BED and its inclusion in the main text of the DSM-5 as a distinct eating disorder, it is expected that BED will become increasingly recognized, diagnosed, and treated by both psychiatrists and primary care physicians. Based on a review of the published literature in PubMed, empirical evidence supports the legitimacy of the duration/frequency criterion for BED and the primary importance of loss of control over eating and marked distress in BED. However, there is a lack of clarity regarding what constitutes a "large amount of food" with reference to binge eating and whether subjective binge eating should be considered in the BED diagnostic criteria. These findings should be considered in light of the fact that only a single database was used in the current review. In addition, although overvaluation of shape/weight is not a diagnostic criterion in the DSM-5, the published literature suggests that it is strongly related to eating pathology severity in individuals with BED and may identify an important subgroup of individuals with BED or serve as an index of severity. Future revisions of the DSM criteria for BED should consider whether to include the undue influence of body shape and weight on self-evaluation as a specifier indicating a BED subtype.

\section{Acknowledgments}

The authors would like to thank Dr Carlos Grilo for his input on the initial drafts of this manuscript and Dr Dawn Eichen for her thoughtful critiques and edits of this manuscript. Under the direction of the authors, writing assistance was provided by Stefan Kolata, $\mathrm{PhD}$ (a former employee of 
Complete Healthcare Communications, LLC [CHC; Chadds Ford, PA]) and Craig Slawecki, PhD (a current employee of $\mathrm{CHC}$ ). Editorial assistance in the form of proofreading, copyediting, and fact-checking was also provided by $\mathrm{CHC}$. The content of this manuscript, the ultimate interpretation, and the decision to submit it for publication in Neuropsychiatric Disease and Treatment were made by the authors. Shire Development LLC (Lexington, MA) provided funding to $\mathrm{CHC}$ for support in writing and editing this manuscript.

\section{Disclosure}

DE Wilfley is a consultant for Shire Pharmaceuticals, LLC. L Citrome has been engaged in collaborative research in the past 36 months with or received consulting or speaking fees from Alexza, Alkermes, Allergan (Actavis, Forest), AstraZeneca, Avanir, Boehringer Ingelheim, Bristol-Myers Squibb, Eli Lilly, Forest, Forum, Genentech, Janssen, Jazz, Lundbeck, Merck, Medivation, Mylan, Novartis, Noven, Otsuka, Pfizer, Reckitt Benckiser, Reviva, Shire, Sunovion, Takeda, Teva, and Valeant. BK Herman is an employee of Shire and holds stock and/or stock options in Shire. The authors report no other conflicts of interest in this work.

\section{References}

1. American Psychiatric Association. Diagnostic and Statistical Manual of Mental Disorders. 5th ed. Washington, DC: American Psychiatric Association; 2013.

2. Stunkard AJ. Eating patterns and obesity. Psychiatr Q. 1959;33: 284-295.

3. American Psychiatric Association. Diagnostic and Statistical Manual of Mental Disorders. 4th ed. Washington, DC: American Psychiatric Association; 1994.

4. Spitzer RL, Stunkard A, Yanovski S, et al. Binge eating disorder should be included in DSM-IV: a reply to Fairburn et al.'s "The classification of recurrent overeating: the binge eating disorder proposal". Int J Eat Disord. 1993;13(2):161-169.

5. Spitzer RL, Devlin MJ, Walsh BT, et al. Binge eating disorder: to be or not to be in the DSM-IV. Int J Eat Disord. 1991;10(6):627-629.

6. Hudson JI, Hiripi E, Pope HG Jr, Kessler RC. The prevalence and correlates of eating disorders in the National Comorbidity Survey Replication. Biol Psychiatry. 2007;61(3):348-358.

7. Kessler RC, Berglund PA, Chiu WT, et al. The prevalence and correlates of binge eating disorder in the World Health Organization World Mental Health Surveys. Biol Psychiatry. 2013;73(9):904-914.

8. Morgan CM, Yanovski SZ, Nguyen TT, et al. Loss of control over eating, adiposity, and psychopathology in overweight children. Int J Eat Disord. 2002;31(4):430-441.

9. Johnson WG, Rohan KJ, Kirk AA. Prevalence and correlates of binge eating in white and African American adolescents. Eat Behav. 2002;3(2):179-189.

10. Grilo CM, Crosby RD, Masheb RM, et al. Overvaluation of shape and weight in binge eating disorder, bulimia nervosa, and sub-threshold bulimia nervosa. Behav Res Ther. 2009;47(8):692-696.

11. Mitchell KS, Neale MC, Bulik CM, Aggen SH, Kendler KS, Mazzeo SE. Binge eating disorder: a symptom-level investigation of genetic and environmental influences on liability. Psychol Med. 2010;40(11): 1899-1906.
12. Niego SH, Pratt EM, Agras WS. Subjective or objective binge: is the distinction valid? Int J Eat Disord. 1997;22(3):291-298.

13. Pratt EM, Niego SH, Agras WS. Does the size of a binge matter? Int $J$ Eat Disord. 1998;24(3):307-312.

14. Palavras MA, Morgan CM, Borges FM, Claudino AM, Hay PJ. An investigation of objective and subjective types of binge eating episodes in a clinical sample of people with co-morbid obesity. J Eat Disord. 2013;1:26.

15. Grilo CM, Hrabosky JI, White MA, Allison KC, Stunkard AJ, Masheb RM. Overvaluation of shape and weight in binge eating disorder and overweight controls: refinement of a diagnostic construct. $J$ Abnorm Psychol. 2008;117(2):414-419.

16. Grilo CM, Masheb RM, White MA. Significance of overvaluation of shape/weight in binge-eating disorder: comparative study with overweight and bulimia nervosa. Obesity (Silver Spring). 2010;18(3): 499-504.

17. Bartholome LT, Peterson RE, Raatz SK, Raymond NC. A comparison of the accuracy of self-reported intake with measured intake of a laboratory overeating episode in overweight and obese women with and without binge eating disorder. Eur J Nutr. 2013;52(1):193-202.

18. Guss JL, Kissileff HR, Devlin MJ, Zimmerli E, Walsh BT. Binge size increases with body mass index in women with binge-eating disorder. Obes Res. 2002;10(10):1021-1029.

19. Yanovski SZ, Leet M, Yanovski JA, et al. Food selection and intake of obese women with binge-eating disorder. Am J Clin Nutr. 1992;56(6): 975-980.

20. Pollert GA, Engel SG, Schreiber-Gregory DN, et al. The role of eating and emotion in binge eating disorder and loss of control eating. Int $J$ Eat Disord. 2013;46(3):233-238.

21. Telch CF, Pratt EM, Niego SH. Obese women with binge eating disorder define the term binge. Int J Eat Disord. 1998;24(3):313-317.

22. White MA, Grilo CM. Diagnostic efficiency of DSM-IV indicators for binge eating episodes. J Consult Clin Psychol. 2011;79(1):75-83.

23. Colles SL, Dixon JB, O'Brien PE. Loss of control is central to psychological disturbance associated with binge eating disorder. Obesity (Silver Spring). 2008;16(3):608-614.

24. Latner JD, Clyne C. The diagnostic validity of the criteria for binge eating disorder. Int J Eat Disord. 2008;41(1):1-14.

25. Nangle DW, Johnson WG, Carr-Nangle RE, Engler LB. Binge eating disorder and the proposed DSM-IV criteria: psychometric analysis of the Questionnaire of Eating and Weight Patterns. Int J Eat Disord. 1994; 16(2):147-157.

26. Coker EL, von Lojewski A, Luscombe GM, Abraham SF. The difficulty in defining binge eating in obese women: how it affects prevalence levels in presurgical bariatric patients. Eat Behav. 2015;17: 130-135.

27. Antoniou M, Tasca GA, Wood J, Bissada H. Binge eating disorder versus overeating: a failure to replicate and common factors in severely obese treatment seeking women. Eat Weight Disord. 2003;8(2):145-149.

28. Kolotkin RL, Westman EC, Ostbye T, Crosby RD, Eisenson HJ, Binks M. Does binge eating disorder impact weight-related quality of life? Obes Res. 2004;12(6):999-1005.

29. Striegel-Moore RH, Dohm FA, Solomon EE, Fairburn CG, Pike KM, Wilfley DE. Subthreshold binge eating disorder. Int J Eat Disord. 2000;27(3):270-278.

30. Grilo CM, White MA, Masheb RM. Significance of overvaluation of shape and weight in an ethnically diverse sample of obese patients with binge-eating disorder in primary care settings. Behav Res Ther. 2012;50(5):298-303.

31. Grilo CM, White MA. A controlled evaluation of the distress criterion for binge eating disorder. J Consult Clin Psychol. 2011;79(4):509-514.

32. Goldschmidt AB, Hilbert A, Manwaring JL, et al. The significance of overvaluation of shape and weight in binge eating disorder. Behav Res Ther. 2010;48(3):187-193.

33. Goldschmidt AB, Le Grange D, Powers P, et al. Eating disorder symptomatology in normal-weight vs obese individuals with binge eating disorder. Obesity (Silver Spring). 2011;19(7):1515-1518. 
34. Franko DL, Becker AE, Thomas JJ, Herzog DB. Cross-ethnic differences in eating disorder symptoms and related distress. Int JEat Disord. 2007;40(2):156-164.

35. American Psychiatric Association. Diagnostic and Statistical Manual of Mental Disorders - Text Revision. 4th ed. Washington, DC: American Psychiatric Association; 2000.

36. Yanovski SZ. Binge eating disorder: current knowledge and future directions. Obes Res. 1993;1(4):306-324.

37. Wilson GT, Sysko R. Frequency of binge eating episodes in bulimia nervosa and binge eating disorder: diagnostic considerations. Int $J$ Eat Disord. 2009;42(7):603-610.

38. Friederich HC, Schild S, Wild B, et al. Treatment outcome in people with subthreshold compared with full-syndrome binge eating disorder. Obesity (Silver Spring). 2007;15(2):283-287.

39. Hudson JI, Coit CE, Lalonde JK, Pope HG Jr. By how much will the proposed new DSM-5 criteria increase the prevalence of binge eating disorder? Int J Eat Disord. 2012;45(1):139-141.

40. Trace SE, Thornton LM, Root TL, et al. Effects of reducing the frequency and duration criteria for binge eating on lifetime prevalence of bulimia nervosa and binge eating disorder: implications for DSM-5. Int J Eat Disord. 2012;45(4):531-536.

41. Hrabosky JI, Masheb RM, White MA, Grilo CM. Overvaluation of shape and weight in binge eating disorder. J Consult Clin Psychol. 2007;75(1):175-180.

42. Grilo CM, White MA, Masheb RM. DSM-IV psychiatric disorder comorbidity and its correlates in binge eating disorder. Int J Eat Disord. 2009;42(3):228-234.

43. Stice E, Marti CN, Rohde P. Prevalence, incidence, impairment, and course of the proposed DSM-5 eating disorder diagnoses in an 8-year prospective community study of young women. J Abnorm Psychol. 2013; 122(2):445-457.

44. Dingemans AE, van Furth EF. Binge eating disorder psychopathology in normal weight and obese individuals. Int J Eat Disord. 2012; 45(1):135-138.

45. Heaner MK, Walsh BT. A history of the identification of the characteristic eating disturbances of bulimia nervosa, binge eating disorder and anorexia nervosa. Appetite. 2013;65:185-188.

46. Walsh BT. The importance of eating behavior in eating disorders. Physiol Behav. 2011;104(4):525-529.

47. Grilo CM, Ivezaj V, White MA. Evaluation of the DSM-5 severity indicator for binge eating disorder in a community sample. Behav Res Ther. 2015;66:72-76.

48. Grilo CM, Ivezaj V, White MA. Evaluation of the DSM-5 severity indicator for binge eating disorder in a clinical sample. Behav Res Ther. 2015;71:110-114.

49. Sandberg RM, Dahl JK, Vedul-Kjelsas E, et al. Health-related quality of life in obese presurgery patients with and without binge eating disorder, and subdiagnostic binge eating disorders. J Obes. 2013;2013: 878310.

50. Fandino J, Moreira RO, Preissler C, et al. Impact of binge eating disorder in the psychopathological profile of obese women. Compr Psychiatry. 2010;51(2):110-114.

51. Barnes RD, White MA, Martino S, Grilo CM. A randomized controlled trial comparing scalable weight loss treatments in primary care. Obesity (Silver Spring). 2014;22(12):2508-2516.

52. Schulz S, Laessle RG. Associations of negative affect and eating behaviour in obese women with and without binge eating disorder. Eat Weight Disord. 2010;15(4):e287-e293.

53. Raymond NC, Bartholome LT, Lee SS, Peterson RE, Raatz SK. A comparison of energy intake and food selection during laboratory binge eating episodes in obese women with and without a binge eating disorder diagnosis. Int J Eat Disord. 2007;40(1):67-71.

54. Hrabosky JI, White MA, Masheb RM, Grilo CM. Physical activity and its correlates in treatment-seeking obese patients with binge eating disorder. Int J Eat Disord. 2007;40(1):72-76.
55. Javaras KN, Laird NM, Reichborn-Kjennerud T, Bulik CM, Pope HG Jr, Hudson JI. Familiality and heritability of binge eating disorder: results of a case-control family study and a twin study. Int $J$ Eat Disord. 2008;41(2):174-179.

56. Hudson JI, Lalonde JK, Berry JM, et al. Binge-eating disorder as a distinct familial phenotype in obese individuals. Arch Gen Psychiatry. 2006;63(3):313-319.

57. Grilo CM. Why no cognitive body image feature such as overvaluation of shape/weight in the binge eating disorder diagnosis? Int J Eat Disord. 2013;46(3):208-211.

58. Eldredge KL, Agras WS. Weight and shape overconcern and emotional eating in binge eating disorder. Int J Eat Disord. 1996;19(1):73-82.

59. Mond JM, Hay PJ, Rodgers B, Owen C. Recurrent binge eating with and without the "undue influence of weight or shape on self-evaluation": implications for the diagnosis of binge eating disorder. Behav Res Ther. 2007;45(5):929-938.

60. Masheb RM, Grilo CM. Prognostic significance of two sub-categorization methods for the treatment of binge eating disorder: negative affect and overvaluation predict, but do not moderate, specific outcomes. Behav Res Ther. 2008;46(4):428-437.

61. Ojserkis R, Sysko R, Goldfein JA, Devlin MJ. Does the overvaluation of shape and weight predict initial symptom severity or treatment outcome among patients with binge eating disorder? Int J Eat Disord. 2012;45(4):603-608.

62. Grilo CM, White MA, Gueorguieva R, Wilson GT, Masheb RM. Predictive significance of the overvaluation of shape/weight in obese patients with binge eating disorder: findings from a randomized controlled trial with 12-month follow-up. Psychol Med. 2013;43(6): $1335-1344$.

63. Reas DL, White MA, Grilo CM. Body checking questionnaire: psychometric properties and clinical correlates in obese men and women with binge eating disorder. Int J Eat Disord. 2006;39(4):326-331.

64. Davis C. The epidemiology and genetics of binge eating disorder (BED). CNS Spectr. 2015;20(6):522-529.

65. Sheehan DV, Herman BK. The psychological and medical factors associated with untreated binge eating disorder. Prim Care Companion CNS Disord. 2015;17(2):doi: 10.4088/PCC.14r01732.

66. Grucza RA, Przybeck TR, Cloninger CR. Prevalence and correlates of binge eating disorder in a community sample. Compr Psychiatry. 2007;48(2):124-131.

67. Grilo CM, White MA, Barnes RD, Masheb RM. Posttraumatic stress disorder in women with binge eating disorder in primary care. J Psychiatr Pract. 2012;18(6):408-412.

68. Yip SW, White MA, Grilo CM, Potenza MN. An exploratory study of clinical measures associated with subsyndromal pathological gambling in patients with binge eating disorder. J Gambl Stud. 2011; 27(2):257-270

69. Udo T, McKee SA, White MA, Masheb RM, Barnes RD, Grilo CM. Sex differences in biopsychosocial correlates of binge eating disorder: a study of treatment-seeking obese adults in primary care setting. Gen Hosp Psychiatry. 2013;35(6):587-591.

70. Barnes RD, Boeka AG, McKenzie KC, et al. Metabolic syndrome in obese patients with binge-eating disorder in primary care clinics: a crosssectional study. Prim Care Companion CNS Disord. 2011;13(2):doi: 10.4088/PCC.10m01050.

71. Webb JB, Applegate KL, Grant JP. A comparative analysis of type 2 diabetes and binge eating disorder in a bariatric sample. Eat Behav. 2011; 12(3):175-181

72. Cremonini F, Camilleri M, Clark MM, et al. Associations among binge eating behavior patterns and gastrointestinal symptoms: a populationbased study. Int J Obes. 2009;33(3):342-353.

73. Johnson JG, Spitzer RL, Williams JB. Health problems, impairment and illnesses associated with bulimia nervosa and binge eating disorder among primary care and obstetric gynaecology patients. Psychol Med. 2001;31(8):1455-1466. 
74. Hudson JI, Lalonde JK, Coit CE, et al. Longitudinal study of the diagnosis of components of the metabolic syndrome in individuals with binge-eating disorder. Am J Clin Nutr. 2010;91(6):1568-1573.

75. Kessler RC, Shahly V, Hudson JI, et al. A comparative analysis of role attainment and impairment in binge-eating disorder and bulimia nervosa: results from the WHO World Mental Health Surveys. Epidemiol Psychiatr Sci. 2014;23(1):27-41.

76. Masheb RM, Grilo CM. Quality of life in patients with binge eating disorder. Eat Weight Disord. 2004;9(3):194-199.

77. Peterson RE, Latendresse SJ, Bartholome LT, Warren CS, Raymond NC Binge eating disorder mediates links between symptoms of depression, anxiety, and caloric intake in overweight and obese women. J Obes. 2012;2012:407103.

78. Ricca V, Castellini G, Lo Sauro C, et al. Correlations between binge eating and emotional eating in a sample of overweight subjects. Appetite. 2009; 53(3):418-421.

79. Masheb RM, Grilo CM. Emotional overeating and its associations with eating disorder psychopathology among overweight patients with binge eating disorder. Int J Eat Disord. 2006;39(2):141-146.

80. Grilo CM, White MA, Barnes RD, Masheb RM. Psychiatric disorder co-morbidity and correlates in an ethnically diverse sample of obese patients with binge eating disorder in primary care settings. Compr Psychiatry. 2013;54(3):209-216.

81. Carrard I, Van der Linden M, Golay A. Comparison of obese and nonobese individuals with binge eating disorder: delicate boundary between binge eating disorder and non-purging bulimia nervosa. Eur Eat Disord Rev. 2012;20(5):350-354.

82. Blomquist KK, Ansell EB, White MA, Masheb RM, Grilo CM. Interpersonal problems and developmental trajectories of binge eating disorder. Compr Psychiatry. 2012;53(8):1088-1095.

83. McElroy SL, Hudson JI, Capece JA, Beyers K, Fisher AC, Rosenthal NR. Topiramate for the treatment of binge eating disorder associated with obesity: a placebo-controlled study. Biol Psychiatry. 2007;61(9): 1039-1048.
84. Sheehan KH, Sheehan DV. Assessing treatment effects in clinical trials with the discan metric of the Sheehan Disability Scale. Int Clin Psychopharmacol. 2008;23(2):70-83.

85. Burton AL, Abbott MJ, Modini M, Touyz S. Psychometric evaluation of self-report measures of binge-eating symptoms and related psychopathology: a systematic review of the literature. Int J Eat Disord. 2016;49(2):125-142.

86. Carrard I, Crepin C, Ceschi G, Golay A, Van der Linden M. Relations between pure dietary and dietary-negative affect subtypes and impulsivity and reinforcement sensitivity in binge eating individuals. Eat Behav 2012;13(1):13-19.

87. Sysko R, Hildebrandt T, Wilson GT, Wilfley DE, Agras WS. Heterogeneity moderates treatment response among patients with binge eating disorder. J Consult Clin Psychol. 2010;78(5):681-690.

88. Kjelsas E, Borsting I, Gudde CB. Antecedents and consequences of binge eating episodes in women with an eating disorder. Eat Weight Disord. 2004;9(1):7-15.

89. Kornstein SG, Kunovac JL, Herman BK, Culpepper L. Recognizing binge eating disorder in the clinical setting. Prim Care Companion CNS Disord. 2016;18(3):doi: 10.4088/PCC.15r01905.

90. Herman BK, Deal LS, DiBenedetti DB, Nelson L, Fehnel SE, Brown TM Development of the 7-item binge eating disorder screener (BEDS-7). Prim Care Companion CNS Disord. 2016;18(2):doi: 10.4088/ PCC.15m01896.

91. Sysko R, Glasofer DR, Hildebrandt T, et al. The eating disorder assessment for DSM-5 (EDA-5): development and validation of a structured interview for feeding and eating disorders. Int J Eat Disord. 2015;48(5):452-463.

92. Cotton MA, Ball C, Robinson P. Four simple questions can help screen for eating disorders. J Gen Intern Med. 2003;18(1):53-56.
Neuropsychiatric Disease and Treatment

\section{Publish your work in this journal}

Neuropsychiatric Disease and Treatment is an international, peerreviewed journal of clinical therapeutics and pharmacology focusing on concise rapid reporting of clinical or pre-clinical studies on a range of neuropsychiatric and neurological disorders. This journal is indexed on PubMed Central, the 'PsycINFO' database and CAS,

\section{Dovepress}

and is the official journal of The International Neuropsychiatric Association (INA). The manuscript management system is completely online and includes a very quick and fair peer-review system, which is all easy to use. Visit http://www.dovepress.com/testimonials.php to read real quotes from published authors. 